\title{
Propagation of Geometric Tolerance Zones in 3D
}

\author{
Song-Hai Zhang ${ }^{1}$, Qi-Hui Zhu ${ }^{2}$, and Ralph R. Martin ${ }^{3}$ \\ 1 Department of Computer Science and Technology, Tsinghua University, Beijing, \\ 100084, China \\ zhangsh@cg.cs.tsinghua.edu.cn \\ 2 Department of Computer and Information Science, University of Pennsylvania, \\ Philadelphia,PA 19104, US \\ qihuizhu@seas . upenn. edu \\ 3 School of Computer Science, Cardiff University, Wales, UK \\ ralph@cs.cf.ac.uk
}

\begin{abstract}
This paper considers error propagation in three dimensional geometric constructions using a geometric approach. First, we present definitions and constructions of tolerance zones for various fundamental elements in Euclidean space. Then, we study in detail the propagation of errors during several geometric computations, including the the distance between two skew lines, reflections, projections, and rotations, and we derive new tolerance zones from the old ones.
\end{abstract}

\section{Introduction}

Computer-aided design and other geometric application areas create a demand for efficient and robust algorithms, which must often deal with imprecisely defined data. There is a need for reliable mathematical foundations of such algorithms, and in particular an understanding of how errors propagate during a chain of calculations with imprecise data.

A commonly used approach to this problem is to use interval arithmetic, which represents imprecise floating point numbers by intervals; some of this work is specific to geometric applications $[1,3,2,7]$. However, in general, interval methods tend to be too conservative, and overestimate the errors produced [6].

An alternative approach to dealing with errors in geometric computations is to use geometric tolerances as proposed by Wallner [9]. In this case, geometric tolerance zones are used, giving a more precise estimate of errors. They also have the important property of geometric invariance under rotations and translations, which interval methods based on coordinates do not [9]. Geometric tolerance computation and propagation have been studied in several papers, considering: errors in construction of geometric fundamental elements $[6,9]$, tolerances of free-form curves [6,9], error propagation in geometric transformations [4], and geometric constraint solving [8]. However, much of this work has concentrated on two-dimensional problems.

In this paper we consider tolerance zone computation and error propagation during various geometric computations in three dimensions, including the 


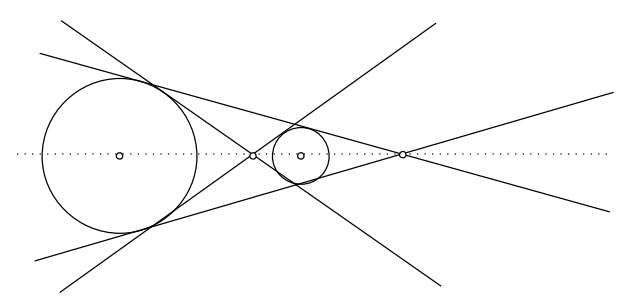

Fig. 1. Tolerance zone of line defined by two points.

distance between two skew lines, and constructions including reflections, projections, and rotations.

\section{Tolerances of fundamental geometric elements}

We start by defining imprecise geometric elements in three dimensions, which are the fundamental items used later in the paper. The simplest assumption to make is that the uncertainty is isotropic, i.e. an imprecisely known point lies somewhere in a sphere centred at its notional position. While this is the simplest model for a tolerance zone, other models may also be appropriate. For example, the uncertainty may be greater in a particular direction, or we may have separate ranges of uncertainty for each axis, leading to initial tolerance zones which are ellipsoidal, or box shaped, for example. In the following, we initially refer to a general 3D tolerance zone $T$ for a point $P$, although later at times we assume more specifically that $T$ is spherical, in order to provide simple results.

In many cases $T$ may naturally be convex: for example, a sphere, ellipsoid, or polyhedron. Minkowski sums are useful when manipulating tolerance zones, as we will explain later. We note that the Minkowski sum of two convex objects is also a convex object-Minkowski sums can be computed much more easily and efficiently for convex objects than for general objects. If a tolerance zone is not naturally convex, it is often convenient to replace it by its convex hull, or some other simple convex shape, for this reason.

We start by defining the basic element, a point associated with a tolerance zone:

Definition 1. A fat point is a $3 D$ point with an associated tolerance zone $T$. If $P$ is the notional position of the point, the fat point is the volume of space given by:

$$
F P(P)=\{Q \mid Q-P \in T\} .
$$

We next define a fat line constructed from two fat points:

Definition 2. A fat line is constructed from two fat points based on $P_{1}, P_{2}$, and is given by

$$
F L\left(P_{1}, P_{2}\right)=\left\{l\left(p_{1}, p_{2}\right) \mid p_{1} \in F P\left(P_{1}\right), p_{2} \in F P\left(P_{2}\right)\right\}
$$

where $l\left(p_{1}, p_{2}\right)$ means the line through points $p_{1}, p_{2}$. 
$F L\left(P_{1}, P_{2}\right)$ denotes the region covered by all lines having one point in $F P\left(P_{1}\right)$ and another point in $F P\left(P_{2}\right)$. Taking a specific case, if $F P\left(P_{1}\right)$ and $F P\left(P_{2}\right)$ are spherical tolerance zones, the resulting volume is bounded, in part, by two cones as shown in Fig. 1; one of the cones may degenerate into a cylinder.

We next define a fat plane constructed from three fat points:

Definition 3. A fat plane is constructed from three fat points based on $P_{1}, P_{2}$, $P_{3}$ and is given by

$$
F P l\left(P_{1}, P_{2}, P_{3}\right)=\left\{\alpha\left(p_{1}, p_{2}, p_{3}\right) \mid p_{1} \in F P\left(P_{1}\right), p_{2} \in F P\left(P_{2}\right), p_{3} \in F P\left(P_{3}\right)\right\}
$$

where $\alpha\left(p_{1}, p_{2}, p_{3}\right)$ is the plane determined by three points.

When $F P\left(P_{1}\right), F P\left(P_{2}\right), F P\left(P_{3}\right)$ are all convex zones, $F P l\left(P_{1}, P_{2}, P_{3}\right)$ can be bounded by eight planes [6], each tangent to the tolerance zone for all three points.

Finally, we define a fat sphere with an associated tolerance zone, based on a fat point, and an uncertain radius. Let $S(P, r)$ be the sphere with centre $P$ and radius $r$. Let $S^{-}(P, r)$ to denote its interior. Then:

Definition 4. A fat sphere defined using the fat point based on $P$, with a radius range of $[r, R]$, is given by

$$
F S(P, r, R)=\bigcup_{p \in F P(P), l \in[r, R]} S(p, l)
$$

If $r$ is sufficiently large, and, for instance, exceeds the radius of $F P(P), F S(P, r, R)$ is a hollow object. Here the radius of $F P(P)$ is defined to be half of the maximum distance between any two points on the boundary. More precisely, $F S=$ $F S_{R}-F S_{r}$. In many cases, for simplicity of calculation, a convex polyhedron will be used as the tolerance zone for a fat point. In such a case, we may write

$$
F S_{R}=\bigcup_{p \in F P(P)} S^{-}(p, R), \quad F S_{r}=\bigcap_{p \in F P(P)} S^{-}(p, r)
$$

Here, $F S_{R}$ is clearly the Minkowski sum of $S^{-}(0, R)$ and $F P(P)$. The expression for $F S_{r}$ can be simplified to

$$
F S_{r}=\bigcap_{p \in V(P)} S^{-}(p, r),
$$

where $V(P)$ denotes the set of vertices of $F P(P)$. F $S_{r}$ could be an empty set, if $r$ is small. We now formally state this as a theorem for the case where the tolerance zone of the fat point is a convex polyhedron, and prove it:

Theorem 1. Given a fat sphere defined as in Definition 4, when $r$ is larger than the diameter of $F P(P), F S(P, r, R)$ can be expressed as $F S(P, r, R)=$ $F S_{R}-F S_{r}$, where

$$
F S_{r}=\bigcap_{p \in V(P)} S^{-}(p, r)
$$




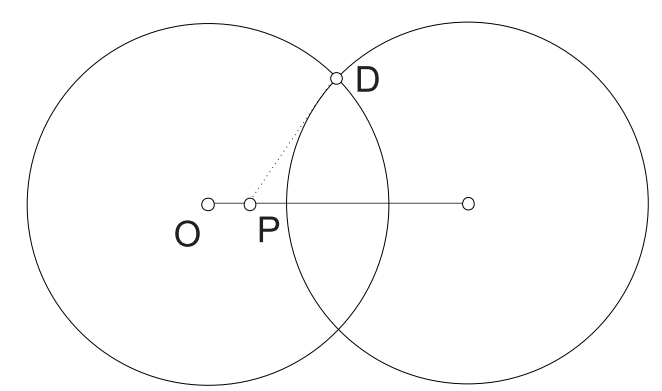

Fig. 2. Illustration of Lemma 1

Proof. It can be readily seen that

$$
\bigcap_{p \in V(P)} S^{-}(p, r) \supseteq \bigcap_{p \in P} S^{-}(p, r)=F S_{r} .
$$

Thus, we have to prove the fact that $\forall p \in P$,

$$
\bigcap_{q \in V(P)} S^{-}(q, r) \subseteq S^{-}(p, r)
$$

and hence the validity of

$$
\bigcap_{p \in V(P)} S^{-}(p, r) \subseteq \bigcap_{p \in P} S^{-}(p, r)=F S_{r} .
$$

We do so using the following three lemmas:

Lemma 1. In two dimensions, let $C^{-}(P, r)$ denote the interior of the circle centred at $P$ with radius $r$. Any point $P$ on the line segment between any two points $P_{1}$ and $P_{2}$ must satisfy $C^{-}(P, r) \supseteq C^{-}\left(P_{1}, r\right) \cap C^{-}\left(P_{2}, r\right)$.

Proof. If $C^{-}\left(P_{1}, r\right) \cap C^{-}\left(P_{2}, r\right)=\emptyset$, then the lemma is obviously correct. Otherwise, we construct a circle centre $O$ with radius $r$ at either end of the line segment $\left(O\right.$ is $P_{1}$ or $\left.P_{2}\right)$. See Fig. 2. $P$ is an arbitrary point on the line segment. Assume $P$ is in the interior of the circle with center $P_{1}$, we can easily see that the intersection of the two circles centered at $P_{1}$ and $P_{2}$ respectively lies within the circle centered at $P$ : distance $P D$ is smaller than distance $O D$, i.e. $P D<r$. By symmetry, the same holds if $P$ is in the interior of the circle with centre $P_{2}$.

Lemma 2. Generalising Lemma 1 to three dimensions for a sphere, $S^{-}(P, r) \supseteq$ $S^{-}\left(P_{1}, r\right) \cap S^{-}\left(P_{2}, r\right)$.

Lemma 3. Let $V(M)$ be the vertex set of a polygon $M$. Given a point $P$ inside the polygon $M$, Then

$$
S^{-}(P, r) \supseteq \bigcap_{Q \in V(M)} S^{-}(Q, r) .
$$




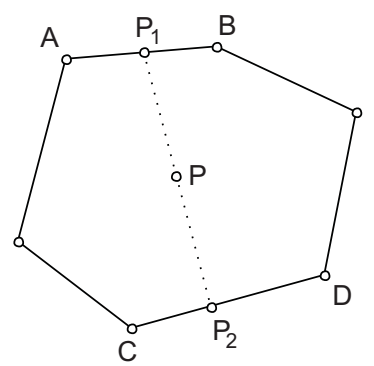

Fig. 3. Illustration of Lemma 3

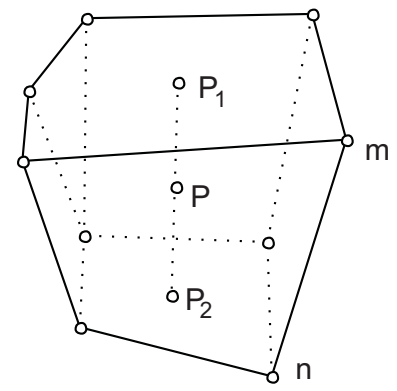

Fig. 4. Convex polyhedron

Proof. Construct some line through $P$ that meets the boundary of the polygon at points $P_{1}, P_{2}$, on edges $A B$ and $C D$, as shown in Fig. 3. According to Lemma 2,

$$
S^{-}(P, r) \supseteq S^{-}\left(P_{1}, r\right) \cap S^{-}\left(P_{2}, r\right)
$$

and

$$
S^{-}\left(P_{1}, r\right) \supseteq S^{-}(A, r) \cap S^{-}(B, r), \quad S^{-}\left(P_{2}, r\right) \supseteq S^{-}(B, r) \cap S^{-}(C, r),
$$

so

$$
S^{-}(P, r) \supseteq S^{-}(A, r) \cap S^{-}(B, r) \cap S^{-}(C, r) \cap S^{-}(D, r) \supseteq \bigcap_{Q \in V(M)} S^{-}(Q, r) .
$$

We now complete the proof of Theorem 1. For a point inside the polyhedron representing the tolerance zone of the fat point, construct some line intersecting the faces of the polyhedron at $P_{1}, P_{2}$, as shown in Fig. 4. Using Lemmas 2 and 3 , the following statements may now be proved in turn:

$$
\begin{aligned}
S^{-}(P, r) & \supseteq S^{-}\left(P_{1}, r\right) \cap S^{-}\left(P_{2}, r\right), \\
S^{-}\left(P_{1}, r\right) \cap S^{-}\left(P_{2}, r\right) & \supseteq \bigcap_{p \in V(m)} S^{-}(p, r) \cap \bigcap_{p \in V(n)} S^{-}(p, r),
\end{aligned}
$$




$$
\bigcap_{p \in V(m)} S^{-}(p, r) \cap \bigcap_{p \in V(n)} S^{-}(p, r) \supseteq \bigcap_{p \in V(M)} S^{-}(p, r),
$$

as required.

We now give an alternative definition of a fat sphere based on four fat points.

Definition 5. The fat sphere generated by four fat points based on $P_{1}, P_{2}, P_{3}$, $P_{4}$ is given by

$$
\begin{aligned}
& F S\left(P_{1}, P_{2}, P_{3}, P_{4}\right)= \\
& \left\{\sigma\left(p_{1}, p_{2}, p_{3}, p_{4}\right) \mid p_{1} \in F P\left(P_{1}\right), p_{2} \in F P\left(P_{2}\right), p_{3} \in F P\left(P_{3}\right), p_{4} \in F P\left(P_{4}\right)\right\}
\end{aligned}
$$

where $\sigma(p, q, r, s)$ is the function which constructs a sphere through four points $p, q, r, s$.

We may now state the following theorem, assuming that the fat points in this case have spherical tolerance zones:

Theorem 2. $\partial F S\left(P_{1}, P_{2}, P_{3}, P_{4}\right) \subseteq \Sigma$ where $\Sigma$ is a set comprising 16 spheres that are each tangent to $F P\left(P_{i}\right), i=1, \ldots, 4$.

Proof. Suppose $P$ is an arbitrary point on the boundary of the fat sphere. Let $\Omega$ be a sphere passing through $P$, determined by 4 points $A, B, C, D$, one from each fat point zone respectively. We will prove that $\Omega$ is tangent to all 4 fat zones. Let $\Omega(A)$ be a sphere passing through $A$ and intersecting the other three fat point zones. Perform a polar transformation with center at $A$, so all spheres passing through $A$ transfrom into planes and spheres not passing through $A$ remain spheres afterwards. After this transformation, $\mathcal{P}\left(F P\left(P_{i}\right)\right)$ are still spheres. $\mathcal{P}(\Omega(A))$ is a plane passing through $A$. In particular, $\mathcal{P}(\Omega(A))$ is the fat plane passing through the three spheres which are the images of three fat points under polar transformation. After polar transformation, if a point is originally interior to a sphere, it remains interiority to the image of the sphere. Points $P$ in $(\Omega(A))$ are boundary points of the fat sphere if under polar transformation, there exists a plane passing through $P^{\prime}$, the image of $P$, which is tangent to images of three spheres. Thus, $\Omega(A)$ is tangent to all the spheres. More precisely, the boundary of the fat sphere is composed of 16 common tangent spheres: each sphere has two types of tangency, so four spheres have 16 different tangent spheres.

\section{Tolerance Zones and Geometric Computations in 3D}

We now consider various geometric computations in three dimensions when performed on objects defined with respect to tolerance zones.

\subsection{Skew line detection and distance computation}

This section considers the issue of deciding whether two fat lines intersect, and the distance between two skew lines, assuming they are based on points with 


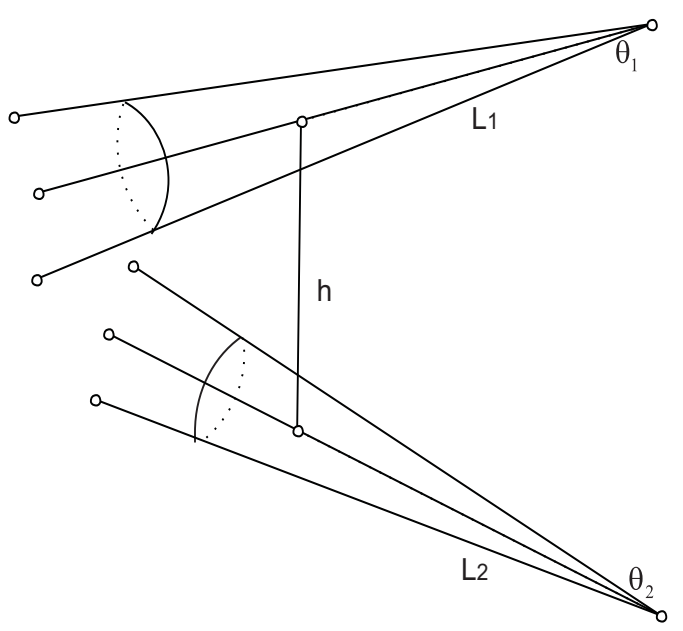

Fig. 5. Distance between two cones

spherical tolerance zones. We combine the detection and distance computation into one algorithm. We assume the two fat lines are skew lines; if their distance is negative, then the lines intersect.

A continuity argument shows that the distance between two skew fat lines may be represented by a single interval, i.e. it can take on any value between a minimum, and a maximum, value.

As explained earlier, a fat line determined by two fat points is bounded by two cones. We compute the distance interval by considering pairs of defining cones, one from each fat line.

Thus, all we must consider is the maximum and minimum distance between two cones. Let $\theta_{1}, \theta_{2}$ denote the semi-angles of the two cones. and let $h$ be the skew distance between their axes (see Figure 5). $O_{1}$ and $O_{2}$ are two origins, and $l_{1}$ and $l_{2}$ are two axes. Suppose points $P_{1}$ and $P_{2}$ lie on the two cones respectively, and the distance between these two points is minimum (or maximum). These points must satisfy:

1. $P_{1}$ and $P_{2}$ lie on the cone surfaces;

2. the line $P_{1} P_{2}$ is normal to the cones at $P_{1}$ and $P_{2}$, so it also passes through axis of each cone. Thus, the angle between line $P_{1} P_{2}$ and $l_{1}$ is $\pi / 2 \pm \theta_{1}$, and the angle between line $P_{1} P_{2}$ and $l_{2}$ is $\pi / 2 \pm \theta_{2}$.

Only lines on the cones need be considered to calculate maximum and minimum distances. Each fat line has two cones, so there are 4 combinations of cones to consider. We call the interval formed from the maximum and minimum distance arising from one combination a partial distance $\left[d_{i}^{\min }, d_{i}^{\max }\right]$. The continuity argument given before shows that the final result $\left[D_{\min }, D_{\max }\right]$ must be the union of these four intervals. The main problem is now to compute $d_{i}^{\text {min }}$ and $d_{i}^{\max }$ for each pair of cones. This calculation can be done as a special case of the 


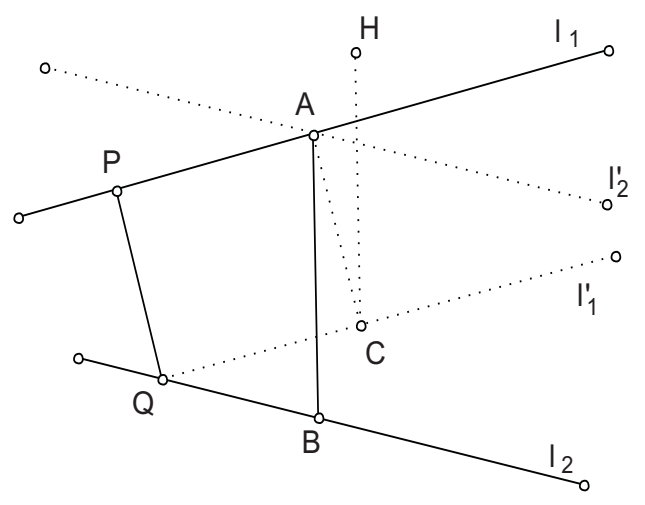

Fig. 6. Distance between two skew lines

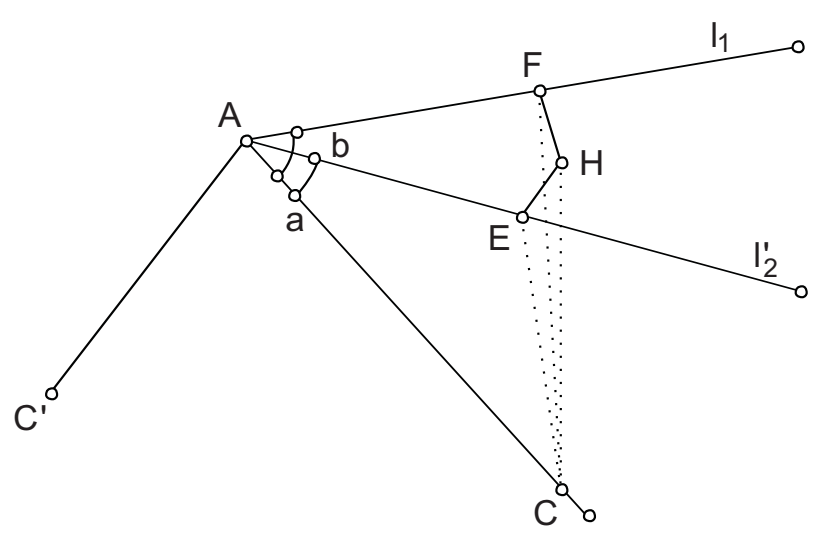

Fig. 7. Two possibilities for $A C$

method given in [5], which considers the distance between a canal surface and a simple surface. Here we give an alternative, direct, approach.

See Figure 6 . The axes of the cones under consideration are $l_{1}, l_{2}$, and their semi-angles are $\theta_{1}, \theta_{2}$. Points $A$ and $B$ are are the points of closest approach of the two axes (the distance between them is $h$ ). Points $P$ and $Q$ are the points where the line $P_{1} P_{2}$ meets each axis $l_{1}, l_{2}$ respectively. Note that the angles between $P Q$ and $l_{1}, l_{2}$ respectively are $\frac{\pi}{2}-\theta_{1}, \frac{\pi}{2}-\theta_{2}$. Construct $l_{1}^{\prime}, l_{2}^{\prime}$ parallel to $l_{1}, l_{2}$, so that $l_{2}^{\prime}$ meets $l_{1}$ in $A$ and $l_{1}^{\prime}$ meets $l_{2}$ in $Q$. Shift $P Q$ to $A C$. $A C$ is now either a minimum or maximum distance between two cones (equal to $P Q$ ). as desired. The distance $C H$, from $C$ to the plane determined by $l_{1}$ and $l_{2}^{\prime}$, is exactly $h$, the extremal distance between the axes.

Figure 7 shows in further detail how to compute $A C$, and the extremal distance. The angles between $A C$ and $l_{1}, l_{2}$ are $\pi / 2 \pm \theta_{1}, \pi / 2 \pm \theta_{2}$ respectively, giving four possible cases to consider for $A C$. Construct the projections $E$ and $F$ of $H$ on $l_{1}$ and $l_{2}^{\prime}$ on the given plane. $C F$ and $F H$, and $C E$ and $E H$ are both 


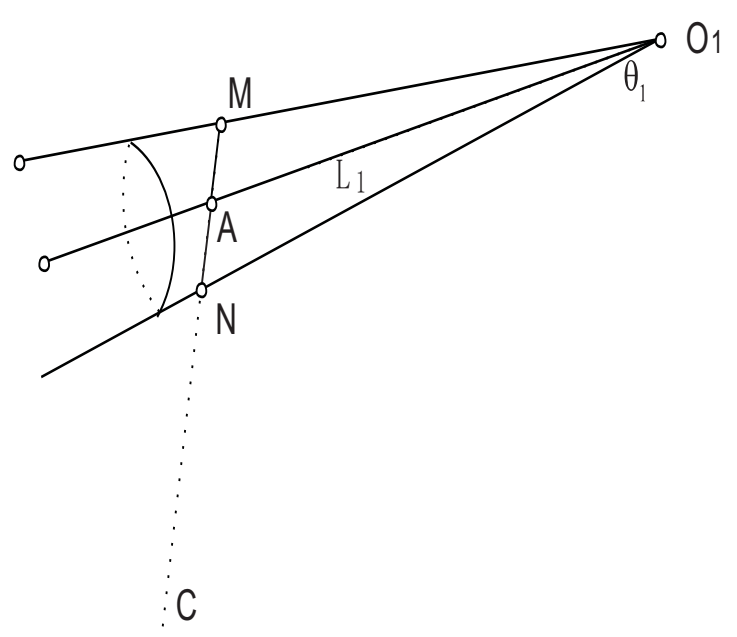

Fig. 8. the Distance inside cones

perpendicular to $l_{1}$, and $l_{2}$ respectively. The following formulae can be obtained, starting from $A C=D, C H=h$, and $\theta$ is the angle between $l_{1}$ and $l_{2}^{\prime}$ :

$$
\begin{gathered}
A E=D \cos a, \quad A F=D \cos b, \quad E F=D \sqrt{\cos ^{2} a+\cos ^{2} b-2 \cos a \cos b \cos \theta}, \\
A H=\frac{D \sqrt{\cos ^{2} a+\cos ^{2} b-2 \cos a \cos b \cos \theta}}{\sin \theta}, \\
h=C H=\sqrt{D^{2}-A H^{2}}=\frac{D \sqrt{\sin ^{2} \theta-\cos ^{2} a-\cos ^{2} b+2 \cos a \cos b \cos \theta}}{\sin \theta}, \\
D=\frac{h \sin \theta}{\sqrt{\sin ^{2} \theta-\cos ^{2} a-\cos ^{2} b+2 \cos a \cos b \cos \theta}},
\end{gathered}
$$

Substituting $a=\pi / 2 \pm \theta_{1}, b=\pi / 2 \pm \theta_{2}$, in which $\theta_{1}, \theta_{2}$ are acute angles, we get

$$
D=\frac{h \sin \theta}{\sqrt{\sin ^{2} \theta-\sin ^{2} \theta_{1}-\sin ^{2} \theta_{2} \pm 2 \sin \theta_{1} \sin \theta_{2} \cos \theta}} .
$$

In addition, the lengths of the line segments inside each cone must be added or subtracted from $D$ to give the overall maximal or minimal distance (see Figure 8). These segments are $A M$ and $A N$ and their lengths should be added to or subtracted from $A C$ : note that $A C$ is perpendicular to $O_{1} M$. We can easily find that

$$
\begin{gathered}
A M=O_{1} A / \sin \theta_{1} \\
A N=O_{1} A\left(\tan 2 \theta_{1} / \cos \theta_{1}-\sin \theta_{1}\right) .
\end{gathered}
$$

Determination of whether addition or subtraction is required depends on the relative positions of $A C$ and the cone axis $l_{1}$.

The distance between two fat lines $\left[D_{\min }, D_{\max }\right]$ can thus be computed by combining four groups of minimum and maximum distances between cones. 


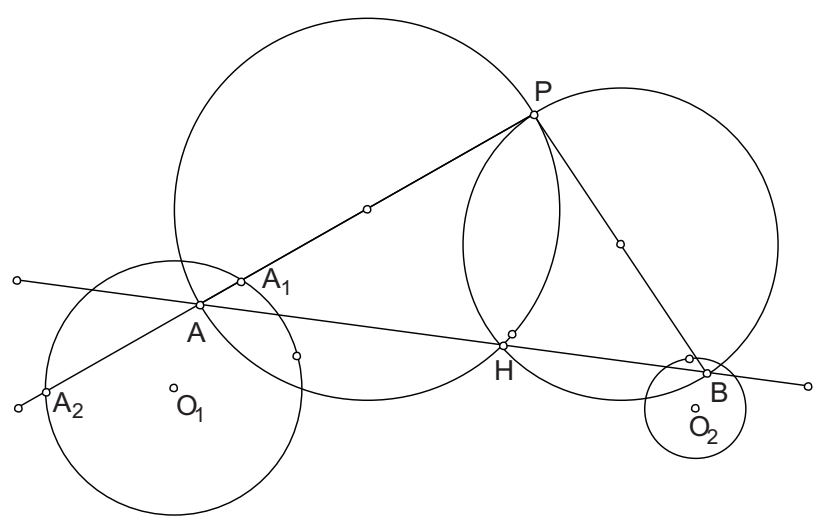

Fig. 9. Illustration of Theorem 3

\subsection{Reflection and line projection in $2 \mathrm{D}$}

In this section we consider how to reflect an exact point in a fat line in $2 \mathrm{D}$, using circular tolerance zones. A closely related problem is that of projecting an exact point onto a fat line in 2D: the projection of a point onto a line is the midpoint of the line joining the point and its reflection. Thus, a solution to either problem gives a solution to the other. Here, we consider $2 \mathrm{D}$ projection of an exact point onto a fat line; we then extend the ideas into 3D space in the next section.

If we wish to project a fat point onto a fat line, this can readily be done if the fat point is represented by a convex polygon. The tolerance zone of the projection can be found by computing the hull of the tolerance zones of the projection of each vertex of the polygon. The case in which the fat point is defined as a circular tolerance zone is more complicated, however, and further work is needed.

We start by giving a theorem concerning projection of an exact point $P$ in a fat line.

Theorem 3. Suppose we are given a $2 \mathrm{D}$ fat line $F L\left(O_{1}, O_{2}\right)$ defined in terms of two fat points with circular tolerance zones $F P\left(O_{i}\right)$, with $O_{i}$ as center, and $r_{i}$ as radius respectively. The boundary of the projection of an exact point $P$ in this fat line can be expressed as a pair of curves $\rho=-l \cos \theta \pm r_{i}, i=1,2$ in polar coordinates, where the origin of polar coordinates is placed at $P$, and $O_{1} P$, $\mathrm{O}_{2} \mathrm{P}$ are taken as $\mathrm{\theta}=0$ axes respectively.

Proof. It can be easily seen that under projection, $P$ goes to a region which is the intersection of two heart-shaped zones ( $H$-zones for short), $H_{1}, H_{2}$ where $H_{i}$ is the set of points $H$ such that $H$ is on the circumference of a circle with diameter $P A$, and $\left.A \in F P\left(O_{i}\right)\right\}$. As Figure 9 shows, $H$ is the projection point corresponding to some definite line joining some point $A$ in $F P\left(O_{1}\right)$ to some point $B$ in $F P\left(O_{2}\right): P H \perp A H$ and $P H \perp B H$. Thus $H$ belongs to both $H_{1}$ and $H_{2}$. We now consider how to calculate these two $H$-zones $H_{1}, H_{2}$. 




Fig. 10. Computation of inner and outer boundaries of $H$-zones

See Figure 10, where $O$ corresponds to either $O_{1}$ or $O_{2}$, i.e. the tolerance zone for either of the fat points. Construct a circle of diameter of $P A$, where $A$ is an arbitrary point inside circle $O$. This circle intersects a line $l$ passing through $P$ at $Q$ with $A Q \perp l$, i.e. $Q$ is the projection of $A$ on $l$. The $H$-zone consists of the envelope of all such circumferences. No point inside circle $O$ can have a corresponding point $Q$ which lies outside the line segment $Q_{1} Q_{2}$, bounded by the tangents to $F P(O)$ as shown in Figure 10. As $l$ rotates through an angle ranging from $-\pi / 2$ to $\pi / 2$, the positions of $Q_{1}$ and $Q_{2}$ sweep out the inner and outer boundaries of the $H$-zone.

If $l$ has an angle given by $\theta$ in polar coordinates with line $P O, \theta \in[-\pi, \pi]$. In polar coordinates $r$ is the radius of circle $O$. Thus, we have

$$
\begin{aligned}
& \overrightarrow{P Q_{1}}=-\overrightarrow{P O} \cos \theta+r, \\
& \overrightarrow{P Q_{2}}=-\overrightarrow{P O} \cos \theta-r .
\end{aligned}
$$

Hence the boundaries of the $H$-zone are $\rho=-\overrightarrow{P O_{i}} \cos \theta \pm r_{i}, i=1,2$.

Having found the projection of a point in a fat line, it is now straightforward to find the corresponding regions for reflection of a point in a fat line. The equations are simply:

$$
\rho=-2 \overrightarrow{P O_{i}} \cos \theta \pm 2 r_{i}, \quad i=1,2 .
$$

\subsection{Reflection and plane projection in $3 \mathrm{D}$}

We now consider projection and reflection of a point in a fat plane in 3D. Theorem 3 can be generalized as follows:

Theorem 4. The tolerance zone formed by projection of a point $P$ onto a fat plane in $3 D$ is surrounded by 6 surfaces with equations in polar coordinates:

$$
\rho=l \cos \theta \cos \varphi \pm r_{i}, \quad i=1, \ldots, 3
$$




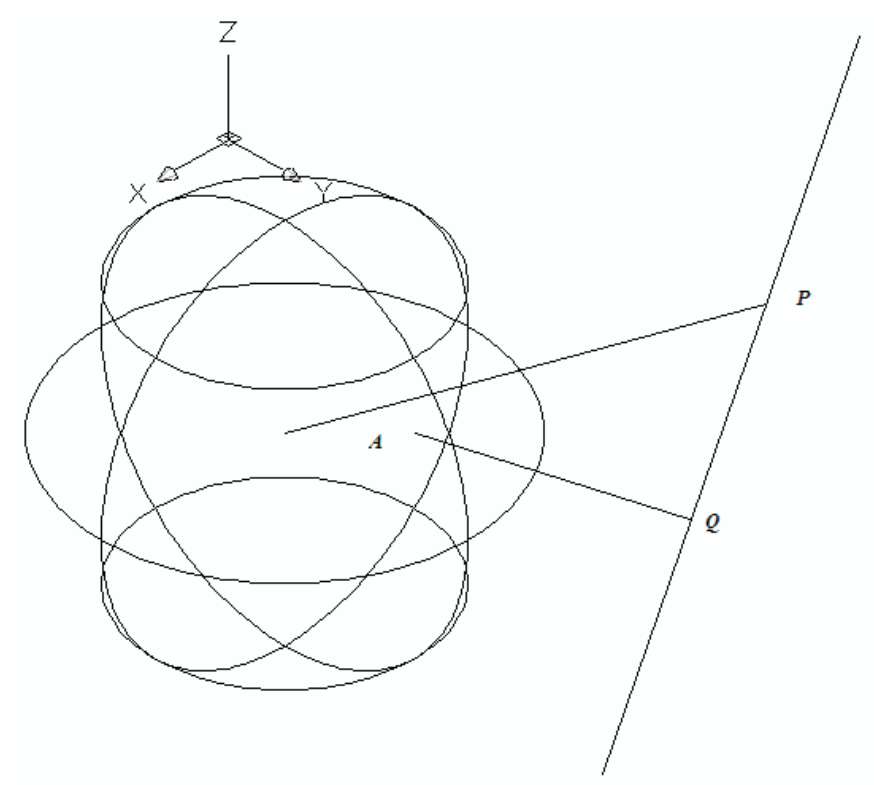

Fig. 11. Tolerance zone for $3 \mathrm{D}$ projection

Proof. Following the ideas in 2D, the tolerance zone is the intersection of three $H$-zones $H_{1}, H_{2}, H_{3}$. To find its boundaries, consider an arbitrary line $l(\theta, \varphi)$, whose spatial orientation is given in spherical polar coordinates by $\theta, \varphi$ (eee Figure 11). The intersection of $H_{i}$ and $l(\theta, \varphi)$ is a segment of $l$. Using the same notation as in the $2 \mathrm{D}$ case, if $Q$ is on this segment, the following inequality holds:

$$
l \cos \theta \cos \varphi-r \leq \overrightarrow{P Q} \leq l \cos \theta \cos \varphi+r
$$

Thus, as the oriented line $l$ takes on all orientations in 3D space, the boundaries of tolerance zone are, following the argument used in 2D:

$$
\rho=2 l \cos \theta \cos \varphi \pm 2 r
$$

\subsection{Rotation}

Finally, we consider rotation of a fat point point $P(x, y, z)$ relative to an origin at $Q$, about an arbitrary axis. The transformation can be described by an orthogonal matrix $R$ :

$$
P^{\prime}-Q=(P-Q) R
$$

where $P^{\prime}$ is the position of $P$ after rotation. 
When there is uncertainty in $Q$, denoted by $\Delta Q, P^{\prime}-Q-\Delta Q=(P-Q-$ $\Delta Q) R$ holds for all possible positions of $Q$. We may rearrange this as

$$
P^{\prime}-Q=(P-Q) R+\Delta Q-\Delta Q R
$$

Consider the term arising due to uncertainty, $\Delta Q-\Delta Q R$. If the rotation angle is $\theta$, the magnitude of this error term is $2 \sin \theta / 2$, and it makes an angle $\pi / 2-\theta / 2$ relative to $\Delta Q$. Thus, $\Delta Q-\Delta Q R$ is obtained by rotation through an angle $\pi / 2-\theta / 2$ and stretching by a ratio of $2 \sin \theta / 2$.

If we now consider the specific case where the uncertainty in $Q$ is a sphere with radius $r$, the uncertainty after rotation is still a sphere, but with a radius $2 r \sin \theta / 2$.

Let us now further suppose that there is an uncertainty $\Delta P$ in the original point $P$. The total uncertainty is now $\Delta P R+\Delta Q-\Delta Q R$. This can be viewed as the Minkowski sum of the uncertainty in $P$ after rotation, with $\Delta Q-\Delta Q R$. If $\Delta P$ is also represented by a sphere independently of $\Delta Q$, the final result is a larger sphere of radius $r_{p}+2 r \sin \theta / 2$, where $r_{p}$ is the size of the spherical tolerance zone of $P$.

\section{Conclusion and future work}

We have provided definitions and constructions for various three dimensional geometric elements with tolerance, including points, lines, planes and spheres. We have then shown how to perform several geometric computations which take these tolerances into account, and provide suitable tolerance zones for the output. These calculations include the distance between two skew lines, and various reflections, projections, and rotations, with spherical tolerance zones. We have also discussed other computations with polyhedral tolerance zones.

Representation of tolerance zone boundaries is an important issue, and still needs further work. Boundaries of tolerance zones need to be restricted to certain particular shapes (e.g. spheres and polyhedra) if algorithms are to be efficiently implemented.

\section{References}

1. S. L. Abrams, W. Cho, C.-Y. Hu, T. Maekawa, N. M. Patrikalakis, E. C. Sherbrooke, and X. Ye. Efficient and reliable methods for rounded-interval arithmetic. Computer-aided Design, 30(8):657-665, 1998.

2. Chun-Yi Hu, Takashi Maekawa, Nicholas M. Patrikalakis, and Xiuzi Ye. Robust interval algorithm for surface intersections. Computer-aided Design, 29(9):617-627, 1997.

3. Chun-Yi Hu, Nicholas M. Patrikalakis, and Xiuzi Ye. Robust interval solid modelling part I: representations. Computer-aided Design, 28(10):807-817, 1996.

4. S.-M. Hu and J. Wallner. Error propagation through geometric transformations. Journal for Geometry and Graphics, 2(8):171-183, 2004. 
5. Ku-Jin Kim. Minimum distance between a canal surface and a simple surface. Computer-Aided Design, 35(10):871-879, 2003.

6. H. Pottmann, B. Odehnal, M. Peternell, J. Wallner, and R. Ait Haddou. On optimal tolerancing in computer-aided design. In Ralph Martin and Wenping Wang, editors, Proceedings of the Conference on Geometric Modeling and Processing (GMP-00), pages 347-363, Los Alamitos, April 10-12 2000. IEEE.

7. S. T. Tuohy, T. Maekawa, G. Shen, and N. M. Patrikalakis. Approximation of measured data with interval B-splines. Computer-Aided Design, 29(11):791-799, November 1997.

8. J. Wallner, H.-P. Schröcker, and S.-M. Hu. Tolerances in geometric constraint problems. Reliable Computing, 3(11):235-251, 2005.

9. Johannes Wallner, Rimvydas Krasauskas, and Helmut Pottmann. Error propagation in geometric constructions. Computer-Aided Design, 32(11):631-641, 2000. 